\title{
Non-stationary random vibration of FE structures subjected to moving loads
}

\author{
F. Lu ${ }^{\mathrm{a}, \mathrm{b}}$, D. Kennedy ${ }^{\mathrm{b}}$, F.W. Williams ${ }^{\mathrm{b}}$ and J.H. Lin ${ }^{\mathrm{a}, *}$

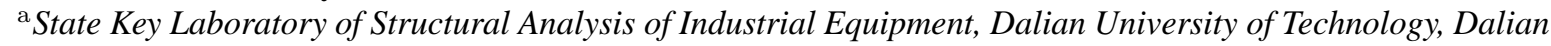 \\ 116023, P.R. China \\ ${ }^{\mathrm{b}}$ Cardiff School of Engineering, Cardiff University, Cardiff CF24 3AA, UK
}

Received 17 November 2006

Revised 2008

\begin{abstract}
An efficient and accurate FEM based method is proposed for studying non-stationary random vibration of structures subjected to moving loads. The loads are assumed to be a stationary process with constant mean value. The non-stationary power spectral densities (PSD) and the time dependent standard deviations of dynamic response are derived by using the pseudo excitation method (PEM) to transform this random excitation problem into a deterministic harmonic excitation one. The precise integration method (PIM) is extended to solve the equation of motion of beams under moving harmonic loads by enhancing the very recent consistent decomposition procedure, in order to simulate the movement of the loads. Six numerical examples are given to show the very high efficiency and accuracy of the method and also to deduce some useful preliminary conclusions from investigation of the dynamic statistical characteristics of a simply supported beam and of a symmetrical three span beam with its centre span unequal to the outer ones.
\end{abstract}

Keywords: Random vibration, moving loads, pseudo excitation method, precise integration method

\section{Introduction}

Dynamic analysis of structures subjected to moving loads is an important research topic in engineering, because it relates to bridges, railways, runways etc. Real road surface irregularities and other unpredictable factors cause traffic loads always to be stochastic, so that in particular random vibration of structures is an important research topic, as follows.

The dynamic response of a simply supported beam subjected to a random moving force with constant speed was studied by Fryba [1,2]. Iwankiewicz and Sniady [3] introduced two influence functions related to the response of beams to obtain expressions for the mean value and variance of beam deflection. Ricciardi [4] assumed the arrival times of multiple moving loads to be random according to a Poisson process and compared his results with those obtained by means of the customary Monte Carlo simulation. Wang and Lin [5] used the method of modal analysis to investigate the random vibration of multi-span Timoshenko beams. Sniady et al. [6] assumed the load to be a set of point forces of random amplitude and stochastic velocity for which the inter-arrival time was a Poisson process and hence they obtained the solution for the probabilistic characteristics of beams by applying Ito integral and differentiation rules. Zibdeh and his co-authors [7-11] were also active in this area as follows: Ref. [7] was concerned with higher order moments of a simply supported elastic beam subjected to a stream of random moving loads of Poissonian type, in both the transient and steady states; Ref. [8] covers beams which were subjected to

\footnotetext{
*Corresponding author: Prof. J.H. Lin, State Key Laboratory of Structural Analysis of Industrial Equipment, Dalian University of Technology, Dalian 116023, P.R. China. Tel.: +86 411 84706337; Fax: +86 411 84706337; E-mail: jhlin@ dlut.edu.cn.
} 
both random moving loads and deterministic axial forces; Ref. [9] investigated the statistical response moments of beams with general boundary conditions and; Refs. $[10,11]$ analyzed random vibration of rotating beams and laminated composite coated beams, respectively. Abu-Hilal [12] considered four boundary conditions and gave the closed-form solution for the variance and the coefficient of variation of the beam deflection.

Due to the efficiency and accuracy limitations of available methods, the above papers used relatively simple models and also usually neglected the cross-correlation between modal responses in order to decrease the computational effort needed by modal analysis. Moreover investigation of the influence of the correlation between loads for multi-load problems is lacking and such simple models do not suffice for the complicated structures which are often built. Therefore FEM is widely used in dynamic analysis of structures subjected to moving loads, particularly for complex problems. However computational difficulties can occur, e.g. the number of degrees of freedom (DOF's) may be excessive and so efficiency and accuracy may be adversely affected. Therefore the purpose of this paper is to develop an efficient and accurate FE method to handle random analysis of structures by using the pseudo excitation (PEM) [13-15] and precise integration (PIM) [16,17] methods.

PEM is a random vibration algorithm, developed from the field of earthquake resistance of bridges, which transforms random excitations into harmonic ones. PIM is an alternative direct integration method which for some cases, e.g. a structure subjected to a fixed harmonic excitation, can give results of essentially computer precision. In the method presented in this paper the FEM is adopted to set up the computational model, with the loads assumed to be stationary random processes of constant mean value. First, PEM is used to transform the loads into harmonic ones and hence the non-stationary responses of structures are derived. Then, PIM is extended to solve the equation of motion of structures subjected to moving harmonic loads by using a consistent decomposition procedure, which has been developed to simulate the movement of the loads. In the numerical examples the proposed method is applied to investigate random vibration of beams. The results show both the advantages of the present method and the dynamic characteristics of single and three span beams. Hence useful conclusions are drawn.

\section{The non-stationary responses of structures subjected to moving loads}

The equation of motion of the FE model of a structure subjected to $l$ moving loads can be written as:

$$
\begin{aligned}
& \mathbf{M} \ddot{\mathbf{u}}+\mathbf{C} \dot{\mathbf{u}}+\mathbf{K u}=\mathbf{D}(t) \mathbf{F}(t) \\
& \mathbf{F}(t)=\left[f_{1}(t), f_{2}(t), \cdots, f_{l}(t)\right]^{\mathrm{T}}
\end{aligned}
$$

where $\mathbf{M}, \mathbf{C}$ and $\mathbf{K}$ are the mass, damping and stiffness matrices; $\mathbf{u}, \dot{\mathbf{u}}$ and $\ddot{\mathbf{u}}$ are the displacement, velocity and acceleration vectors; $\mathbf{D}(t)$ is a time-dependent matrix which represents the distribution of the moving loads; and $\mathbf{F}(t)$ is the random excitation vector with each of its elements $f_{i}(t) \quad(i=1,2, \cdots l)$ assumed to be a random stationary process with constant mean value $\bar{f}_{i}$.

Generally Eq. (1) has many DOF's and so mode superposition is used to reduce the computational effort. Thus the solution of Eq. (1) can be expressed in the Duhamel integration form:

$$
\begin{aligned}
& \mathbf{u}(t)=\sum_{j=1}^{q} \phi_{j} \int_{0}^{t} h_{j}(t-\tau) \gamma_{j}(\tau) \mathbf{F}(\tau) d \tau \\
& \gamma_{j}(t)=\phi_{j}^{\mathrm{T}} \mathbf{D}(t)
\end{aligned}
$$

where: $\phi_{j}(j=1,2, \cdots, q)$ is the $j$ th mode of the structure; $h_{j}(t)$ is the corresponding impulse function; and $\gamma_{j}(t)$ is the mode participation coefficient vector. Applying the expectation operator $E[\cdot]$ to $\mathbf{u}(t)$ gives:

$$
\begin{aligned}
& E[\mathbf{u}(t)]=\overline{\mathbf{u}}(t)=\sum_{j=1}^{q} \phi_{j} \int_{0}^{t} h_{j}(t-\tau) \gamma_{j}(\tau) E[\mathbf{F}] d \tau \\
& E[\mathbf{F}]=\left[\bar{f}_{1}, \bar{f}_{2}, \cdots, \bar{f}_{l}\right]^{\mathrm{T}}
\end{aligned}
$$


where $\overline{\mathbf{u}}(t)$ is the mean value vector. By multiplying $\mathbf{u}(t)-\overline{\mathbf{u}}(t)$ by itself and operating $E[\cdot]$, the auto-variance matrix $\mathbf{R}_{u u}(t, t)$ can be obtained:

$$
\begin{aligned}
& \mathbf{R}_{u u}(t, t)=E\left[\{\mathbf{u}(t)-\overline{\mathbf{u}}(t)\}\{\mathbf{u}(t)-\overline{\mathbf{u}}(t)\}^{\mathrm{T}}\right]=\sum_{j=1}^{q} \sum_{k=1}^{q} \mathbf{R}_{\varphi_{i} \varphi_{j}}(t, t) \\
& \mathbf{R}_{\varphi_{i} \varphi_{j}}(t, t)=\phi_{j} \phi_{k}^{\mathrm{T}} \int_{0}^{t} \int_{0}^{t} h_{j}\left(t-\tau_{1}\right) \gamma_{j}\left(\tau_{1}\right) \mathbf{R}_{F F}(\Delta) \gamma_{k}^{\mathrm{T}}\left(\tau_{2}\right) h_{k}\left(t-\tau_{2}\right) d \tau_{1} d \tau_{2} \\
& \mathbf{R}_{F F}(\Delta)=\left[\begin{array}{ll}
R_{f_{1} f_{1}}(\Delta) \cdots R_{f_{l} f_{f}}(\Delta) \\
\vdots & \ddots \vdots \\
R_{f_{l} f_{1}}(\Delta) \cdots R_{f_{l} f_{l}}(\Delta)
\end{array}\right] ; \quad \Delta=\tau_{2}-\tau_{1}
\end{aligned}
$$

where: $\mathrm{T}$ denotes transformation; $\tau_{1}$ and $\tau_{2}$ are the values of $\tau$ for the two integrations; $\mathbf{R}_{\varphi_{i} \varphi_{j}}(t, t)$ is the auto (cross)-variance matrix of modal responses; $\mathbf{R}_{F F}(\Delta)$ is the covariance matrix of $\mathbf{F}(t)$; and $R_{f_{m} f_{n}}(\Delta)$ is the auto (cross)-covariance function of $f_{i}(t)$.

$\overline{\mathbf{u}}(t)$ and $\mathbf{R}_{u u}(t, t)$ are the most important statistical characteristics of dynamic response. From Eq. (3) it can be seen that in fact $\overline{\mathbf{u}}(t)$ is the mean response of structures under the $l$ deterministic moving constant loads and so can be computed by one of many available methods. Therefore the present paper is focused on solving to find $\mathbf{R}_{u u}(t, t)$, noting that Eq. (4) contains both a double summation and a double integration and so involves quite difficult computation. Sometimes some of the associated computation time is saved by neglecting the cross-correlation between the modal responses [1,2,11,12], i.e. by assuming that $\mathbf{R}_{\varphi_{i} \varphi_{j}}(t, t)=0$ when $i \neq j$. In contrast, in the present paper this cross-correlation is included and $\mathbf{R}_{u u}(t, t)$ is computed efficiently and accurately by using PEM.

\section{Non-stationary PSD and time-dependent standard deviation of structures found by PEM}

This section gives in detail the non-stationary response on the assumption that all loads are fully correlated, i.e. all loads originate from the same excitation source. This assumption is reasonable in some cases. For example, the loads on the wheels on one side of a train are all caused by the same randomly irregular track profile and so are fully correlated but with a time lag difference. Hence $\mathbf{F}(t)$ can be expressed as:

$$
\mathbf{F}(t)=\left[a_{1} f\left(t-t_{1}\right), a_{2} f\left(t-t_{2}\right), \cdots, a_{l} f\left(t-t_{l}\right)\right]^{\mathrm{T}}
$$

where: $a_{i}(i=1,2, \cdots, l)$ represents the intensity of each load; $t_{i}$ is its time lag and; $f(t)$ is the original random excitation.

If $S_{f f}(\omega)$ is the PSD function of $f(t)$, then the excitation PSD matrix $\mathbf{S}_{F F}(\omega)$ is:

$$
\mathbf{S}_{F F}(\omega)=\left[\begin{array}{cccc}
a_{1}^{2} & a_{1} a_{2} \mathrm{e}^{i \omega\left(t_{1}-t_{2}\right)} & \cdots & a_{1} a_{l} \mathrm{e}^{i \omega\left(t_{1}-t_{l}\right)} \\
a_{2} a_{1} \mathrm{e}^{i \omega\left(t_{2}-t_{1}\right)} & a_{2}^{2} & \cdots & a_{2} a_{l} \mathrm{e}^{i \omega\left(t_{2}-t_{l}\right)} \\
\vdots & \vdots & & \vdots \\
a_{l} a_{1} \mathrm{e}^{i \omega\left(t_{l}-t_{1}\right)} & a_{l} a_{2} \mathrm{e}^{i \omega\left(t_{l}-t_{2}\right)} & \cdots & a_{l}^{2}
\end{array}\right] S_{f f}(\omega)
$$

Using PEM this can be rewritten as:

$$
\begin{aligned}
& \mathbf{S}_{F F}(\omega)=\mathbf{y}^{*} \mathbf{y}^{\mathrm{T}} \\
& \mathbf{y}=\left[a_{1} e^{-i \omega t_{1}}, a_{2} e^{-i \omega t_{2}}, \cdots, a_{l} e^{-i \omega t_{l}}\right]^{\mathrm{T}} \sqrt{S_{f f}(\omega)}
\end{aligned}
$$

where the asterisk denotes complex conjugate. Then using the Wiener-Khintchine theorem gives:

$$
\mathbf{R}_{F F}(\Delta)=\int_{-\infty}^{+\infty} \mathbf{S}_{F F}(\omega) e^{i \omega \Delta} d \omega
$$


Hence substituting Eqs (7) and (8) into Eq. (4) gives:

$$
\begin{aligned}
& \mathbf{R}_{u u}(t)=\int_{-\infty}^{+\infty} \mathbf{S}_{u u}(t, \omega) d \omega \\
& \mathbf{S}_{u u}(t, \omega)=\mathbf{I}^{*}(t, \omega) \mathbf{I}^{\mathrm{T}}(t, \omega) \\
& \mathbf{I}(t, \omega)=\sum_{j=1}^{q} \phi_{j} \int_{0}^{t} h_{j}(t-\tau) \gamma_{j}(\tau) \mathbf{y} e^{i \omega \tau} d \tau
\end{aligned}
$$

Obviously $\mathbf{S}_{u u}(t, \omega)$ can be defined as a non-stationary PSD matrix, which represents the distribution of responses in the frequency domain at time $t$, and $\mathbf{I}(t, \omega)$ is the response of the structure caused by the moving harmonic loads $\mathbf{y} e^{i \omega t}$. The key step of the above derivation is changing the order of summations and integrations in Eq. (4), because this enables the cross-correlation between the modal responses to be considered without extra computational effort.

When computing with this method the frequency domain must be discrete and so the accuracy of $\mathbf{R}_{u u}(t)$ depends on the number of harmonic terms used. Convergence occurs as finer and finer frequency domain meshes are adopted, as illustrated by the examples given later.

The basic idea of PEM is to transform random excitation into harmonic functions and the procedures of the random analysis method of the present paper can be summarized as:

Step 1. Discretise the frequency domain and specify the number of harmonic terms.

Step 2. Constitute the pseudo harmonic loads $\mathbf{y} e^{i \omega t}$ corresponding to one of the harmonic frequency terms, see Eq. (7b).

Step 3. Solve for the pseudo response $\mathbf{I}(t, \omega)$, see Eq. (9c), which will be obtained very efficiently by using PIM, see Section 4 below.

Step 4. Multiply $\mathbf{I}(t, \omega)$ by itself to obtain the non-stationary PSD $\mathbf{S}_{u u}(t, \omega)$, see Eq. (9b).

Step 5. If $\mathbf{S}_{u u}(t, \omega)$ has now been obtained for all of the harmonic frequency terms, go to Step 6. Otherwise, go back to Step 2.

Step 6. Compute $\mathbf{R}_{u u}(t)$ by integrating the above $\mathbf{S}_{u u}(t, \omega)$ in the frequency domain, see Eq. (9a).

Load correlation other than fully correlated could also be handled efficiently by PEM by performing the steps described in the previous paragraph, but results are given in this paper only for fully correlated cases and for cases when there is no correlation, i.e. the loads are completely independent of each other, so that the response can be computed by superposing responses for each load acting alone.

\section{PIM solution of the equation of motion of structures subjected to moving loads}

Because PEM is used, see the previous section, it is only necessary to compute the response of structures subjected to moving harmonic loads. Due to its low efficiency Duhamel integration is seldom used for such computations and so instead direct integration methods are adopted. These usually require the magnitude and position of loads to be invariant in each integration step, so that a very small step size is needed to ensure sufficient precision, particularly for rapidly varying loads. This is inefficient and particularly wasteful for random analysis. This shortcoming is overcome in the present paper by extending PIM to solve the equations, using a consistent decomposition procedure to simulate the movement of the loads. This consistent decomposition procedure was developed very recently [18] and was used only with deterministic loads which vary harmonically with constant amplitude. However, because PEM enables the solution of the random force problem to be obtained by considering only harmonically varying forces, the previous consistent decomposition method [18] is applicable to the present paper without modification. However, the detail of modal superposition was omitted in the previous paper and so for completeness it has been added in the re-derivation of the consistent decomposition procedure given in the rest of this section. 


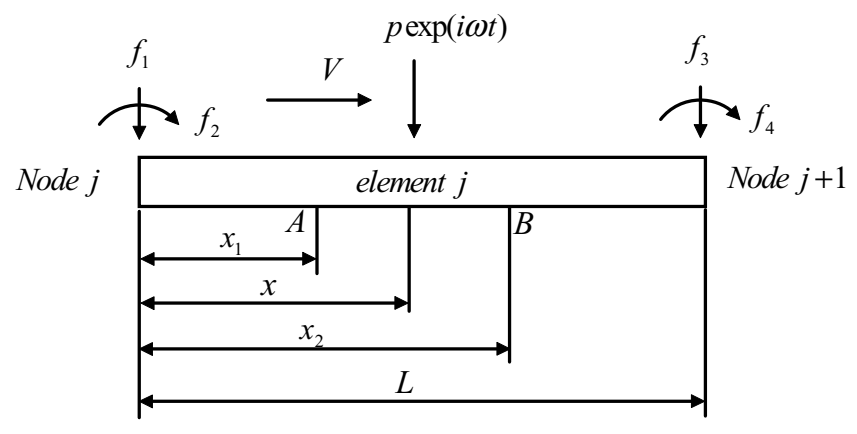

Fig. 1. The consistent decomposition procedure under condition I.

\subsection{Consistent decomposition procedure}

The function of the consistent decomposition procedure is to form the matrix $\mathbf{D}(t)$ in Eq. (1) by superposition of the components yielded by each of the $l$ single moving loads $p \exp (i \omega t)$. For convenience it is assumed that Bernoulli-Euler beam elements are used. In a chosen time step $\left[t_{k}, t_{k}+\Delta t\right]$ the load $p \exp (i \omega t)$ moves from point $\mathrm{A}$ to point B with constant velocity $V$, where $t_{k}$ is the start time of the $k$ th integration step and $\Delta t$ is the time increment. When forming $\mathbf{D}(t)$, the two conditions I and II described in the next two paragraphs are possible, where: (I)A and $\mathrm{B}$ lie in the same element; (II)A and B lie in different elements.

(I) Points A and B lie in the same element, see Fig. 1. The length of the element is $L$ and the distances from node $j$ to points $\mathrm{A}$ and $\mathrm{B}$ and to the location of the load at time $t_{k}+\tau$ are, respectively, $x_{1}, x_{2}$ and $x$. Because the velocity $V$ is constant it follows that:

$$
x=x_{1}+\tau\left(x_{2}-x_{1}\right) / \Delta t ; \quad \xi=\xi_{1}+\tau\left(\xi_{2}-\xi_{1}\right) / \Delta t ; \quad x \in\left[x_{1}, x_{2}\right] ; \quad \tau \in[0, \Delta t]
$$

where $\xi=x / L$ is the dimensionless distance. In FEM, all the variables are replaced by equivalent variables at nodes based on shape functions. So at time $t_{k}+\tau$ the equivalent nodal forces $f_{i}(t)$ are

$$
f_{1}(t)=N_{1}(\xi) p e^{i \omega t} ; \quad f_{2}(t)=N_{2}(\xi) p e^{i \omega t} ; \quad f_{3}(t)=N_{3}(\xi) p e^{i \omega t} ; \quad f_{4}(t)=N_{4}(\xi) p e^{i \omega t}
$$

in which the $N_{i}(\xi),(i=1,2,3,4)$ are the Bernoulli-Euler beam shape functions.

$$
N_{1}=1-3 \xi^{2}+2 \xi^{3}, \quad N_{2}=\left(\xi-2 \xi^{2}+\xi^{3}\right) L, \quad N_{3}=3 \xi^{2}-2 \xi^{3}, \quad N_{4}=\left(-\xi^{2}+\xi^{3}\right) L
$$

Substituting Eqs (10) and (12) into Eq. (11) and forming $\mathbf{D}(t)$ from $f_{i}(t)$ gives:

$$
\begin{aligned}
& \mathbf{D}(t)=\mathbf{a}_{0}+\mathbf{a}_{1} \tau+\mathbf{a}_{2} \tau^{2}+\mathbf{a}_{3} \tau^{3} \\
& \mathbf{a}_{0}=\left\{0,0, \ldots, b_{1}, b_{2}, b_{3}, b_{4}, \ldots, 0,0\right\}^{\mathrm{T}} ; \quad \mathbf{a}_{1}=\left\{0,0, \ldots, b_{5}, b_{6}, b_{7}, b_{8}, \ldots, 0,0\right\}^{\mathrm{T}} \\
& \mathbf{a}_{2}=\left\{0,0, \ldots, b_{9}, b_{10}, b_{11}, b_{12}, \ldots, 0,0\right\}^{\mathrm{T}} ; \quad \mathbf{a}_{3}=\left\{0,0, \ldots, b_{13}, b_{14}, b_{15}, b_{16}, \ldots, 0,0\right\}^{\mathrm{T}} \\
& b_{1}=1-3 \xi_{1}^{2}+2 \xi_{1}^{3} ; \quad b_{2}=L\left(\xi_{1}-2 \xi_{1}^{2}+\xi_{1}^{3}\right) ; \quad b_{3}=1-b_{1} ; \quad b_{4}=L\left(\xi_{1}^{3}-\xi_{1}^{2}\right) \\
& b_{5}=6\left(\xi_{1}^{2}-\xi_{1}\right)\left(\xi_{2}-\xi_{1}\right) / \Delta t ; \quad b_{6}=L\left(\xi_{2}-\xi_{1}\right)\left(1-4 \xi_{1}+3 \xi_{1}^{2}\right) / \Delta t \\
& b_{7}=-b_{5} ; \quad b_{8}=L\left(\xi_{2}-\xi_{1}\right)\left(3 \xi_{1}^{2}-2 \xi_{1}\right) / \Delta t ; \quad b_{9}=\left(6 \xi_{1}-3\right)\left(\xi_{2}-\xi_{1}\right)^{2} / \Delta t^{2} \\
& b_{10}=L\left(\xi_{2}-\xi_{1}\right)^{2}\left(3 \xi_{1}-2\right) / \Delta t^{2} ; \quad b_{11}=-b_{9} ; \quad b_{12}=L\left(\xi_{2}-\xi_{1}\right)^{2}\left(3 \xi_{1}-1\right) / \Delta t^{2} \\
& b_{13}=2\left(\xi_{2}-\xi_{1}\right)^{3} / \Delta t^{3} ; \quad b_{14}=L\left(\xi_{2}-\xi_{1}\right)^{3} / \Delta t^{3} ; \quad b_{15}=-b_{13} ; \quad b_{16}=b_{14}
\end{aligned}
$$

(II) Fig. 2 and its stages (a)-(d) described below show how $\mathbf{D}(t)$ is formed when points A and B lie in different elements.

(a) In an integration step, the load moves from A to Bwhich belongs to another element, i.e. the load crosses boundaries between elements at least once.

(b) Constitute a virtual element with points A and B as its nodes, and let the load move along it, see Fig. 2(b).

(c) Obtain the equivalent end forces $f_{i}(t)$ for the virtual element, see Fig. 1, in the same way as for condition I, so that setting $\xi_{1}=0$ and $\xi_{2}=1$ in Eq. (13) gives: 


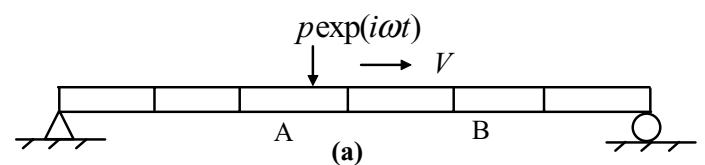

(a)

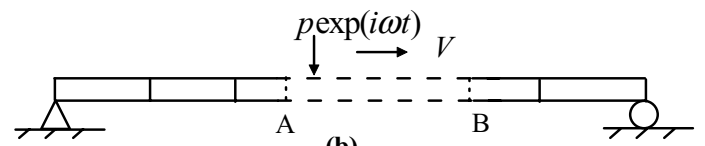

(b)

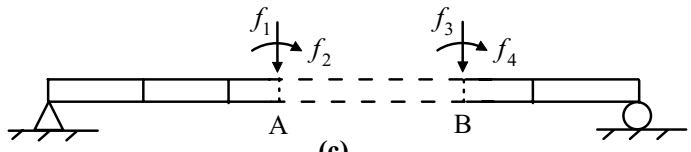

(c)

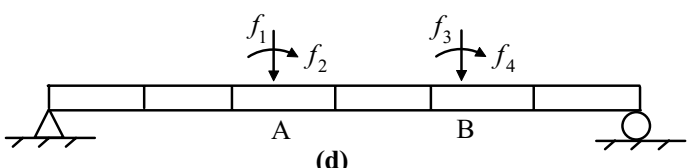

(d)

Fig. 2. The consistent decomposition procedure under condition II. The dashed lines indicate a virtual element.

$$
\begin{aligned}
& f_{1}(t)=\left(c_{1}+c_{2} \tau+c_{3} \tau^{2}+c_{4} \tau^{3}\right) p e^{i \omega t} ; \quad f_{2}(t)=\left(c_{5}+c_{6} \tau+c_{7} \tau^{2}+c_{8} \tau^{3}\right) p e^{i \omega t} \\
& f_{3}(t)=\left(c_{9}+c_{10} \tau+c_{11} \tau^{2}+c_{12} \tau^{3}\right) p e^{i \omega t} ; \quad f_{4}(t)=\left(c_{13}+c_{14} \tau+c_{15} \tau^{2}+c_{16} \tau^{3}\right) p e^{i \omega t} \\
& c_{1}=1 ; \quad c_{2}=0 ; \quad c_{3}=-3 \eta^{2} ; \quad c_{4}=2 \eta^{3} ; \\
& c_{5}=0 ; \quad c_{6}=V ; \quad c_{7}=-2 \eta V ; \quad c_{8}=\eta^{2} V ; \\
& c_{9}=0 ; \quad c_{10}=0 ; \quad c_{11}=3 \eta^{2} ; \quad c_{12}=-2 \eta^{3} ; \\
& c_{13}=0 ; \quad c_{14}=0 ; \quad c_{15}=-\eta V ; \quad c_{16}=\eta^{2} V ; \\
& \quad \eta=1 / \Delta t ;
\end{aligned}
$$

(d) Derive the equivalent forces of the actual elements in which A and B lie (see Fig. 2(d)) from these $f_{i}(t)$ by standard FEM procedures, to obtain $\mathbf{D}(t)$ in a form identical to that of Eq. (13).

The vector $\mathbf{D}(t)$ derived under condition I is exact in FEM terms, whereas that derived under condition II is approximate. Generally, in one integration process condition I will apply for some steps and condition II for others.

For other kinds of beam or plate elements, $\mathbf{D}(t)$ can be formed by a similar procedure to that given above, because FEM shape functions are polynomial, so that $\mathbf{D}(t)$ usually has a similar form to that of Eq. (13).

\subsection{The precise integration expression}

The equation of motion of a structure with $l$ harmonic loads is:

$$
\mathbf{M} \ddot{\mathbf{u}}+\mathbf{C} \dot{\mathbf{u}}+\mathbf{K u}=\sum_{m=1}^{l}\left(\mathbf{e}_{m 0}+\mathbf{e}_{m 1} \tau+\mathbf{e}_{m 2} \tau^{2}+\mathbf{e}_{m 3} \tau^{3}\right) \exp \left[i \omega\left(t_{k}+\tau\right)\right]
$$

where the $\mathbf{e}_{m n}$ are vectors for the $m$ th load and are readily obtained from the $\mathbf{a}_{n}(n=0,1,2,3)$ in Eq. (13), or their equivalent as described beneath Eq. (14). When mode superposition is used, Eq. (15) reduces into $q$ independent equations, which are written in state space, as:

$$
\begin{aligned}
& \dot{\mathbf{v}}_{j}=\mathbf{H}_{j} \mathbf{v}_{j}+\mathbf{r}_{j}(t) \quad j=1,2, \cdots q \\
& \mathbf{H}_{j}=\left[\begin{array}{ll}
0 & 1 \\
-\theta_{j}^{2}-2 \xi_{j} \theta_{j}
\end{array}\right], \quad \mathbf{v}_{j}=\left\{\begin{array}{l}
z_{j} \\
\dot{z}_{j}
\end{array}\right\}, \quad \mathbf{r}_{j}(t)=\left(\mathbf{r}_{j 0}+\mathbf{r}_{j 1} \tau+\mathbf{r}_{j 2} \tau^{2}+\mathbf{r}_{j 3} \tau^{3}\right) \exp (i \omega \tau) \\
& \mathbf{r}_{j n}=\left[\begin{array}{ll}
0, \gamma_{j n}
\end{array}\right]^{\mathrm{T}} \exp \left(i \omega t_{k}\right) ; \quad \gamma_{j n}=\phi_{j}^{\mathrm{T}} \sum_{\mathrm{m}=1}^{1} \mathbf{e}_{m n} ; \quad n=0,1,2,3
\end{aligned}
$$




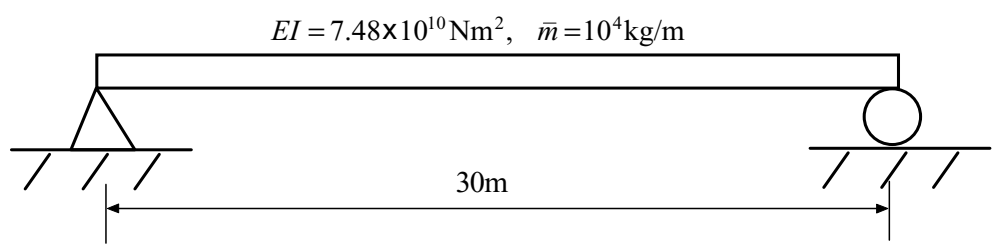

Fig. 3. The simply supported beam.

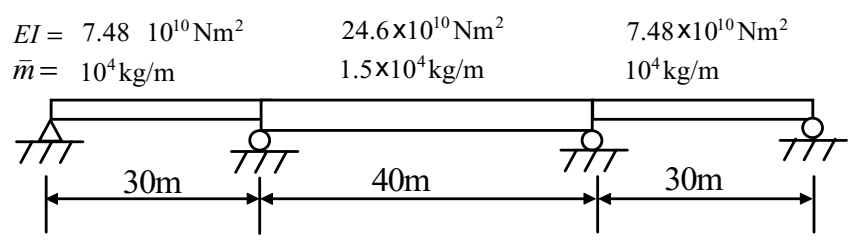

Fig. 4. The three span non-uniform beam, showing $E I$ and $\bar{m}$ for each of its uniform spans.

where: $\theta_{j}$ is the $j$ th natural frequency of the structure; $\phi_{j}, \xi_{j}$ and $z_{j}$ are the corresponding mode, damping ratio and modal response and; $\mathbf{v}_{j}$ is the state space vector. It is assumed that the state space vector $\mathbf{v}_{j}\left(t_{k}\right)$ at time $t_{k}$ is known, so that the state space vector $\mathbf{v}_{j}\left(t_{k+1}\right)$ at time $t_{k+1}=t_{k}+\Delta t$ is $[16,17]$

$$
\mathbf{v}_{j}\left(t_{k+1}\right)=\mathbf{T}_{j}(\Delta t)\left[\mathbf{v}_{j}\left(t_{k}\right)-\mathbf{v}_{j p}\left(t_{k}\right)\right]+\mathbf{v}_{j p}\left(t_{k+1}\right) \quad \mathbf{T}_{j}(\Delta t)=\exp \left(\mathbf{H}_{j} \Delta t\right)
$$

where: $\mathbf{T}_{j}(\Delta t)$ is an exponential matrix, computed in the usual way [16,17] and; $\mathbf{v} j p(t)$ is the particular vector which can be assumed to have identical form to $\mathbf{r}_{j}(t)$, i.e.

$$
\mathbf{v}_{j p}(t)=\left(\mathbf{k}_{j 0}+\mathbf{k}_{j 1} \tau+\mathbf{k}_{j 2} \tau^{2}+\mathbf{k}_{j 3} \tau^{3}\right) \exp (i \omega \tau) ; \quad \tau= \begin{cases}0, & t=t_{k} \\ \tau, & t=t_{k+1}\end{cases}
$$

where $\mathbf{k}_{j n}, \quad(n=0,1,2,3)$ are coefficient vectors. Substituting $\mathbf{r}_{j}(t)$ and $\mathbf{v}_{j p}(t)$ into Eq. (16) and equating the coefficient vectors on the two sides gives

$$
\begin{aligned}
& \mathbf{J}_{j}=-(\mathbf{H}-i \omega \mathbf{I})^{-1} \\
& \mathbf{k}_{j 0}=\mathbf{J}_{j}\left(\mathbf{r}_{j 0}-\mathbf{k}_{j 1}\right) ; \quad \mathbf{k}_{j 1}=\mathbf{J}_{j}\left(\mathbf{r}_{j 1}-2 \mathbf{k}_{j 2}\right) ; \quad \mathbf{k}_{j 2}=\mathbf{J}_{j}\left(\mathbf{r}_{j 2}-3 \mathbf{k}_{j 3}\right) ; \quad \mathbf{k}_{j 3}=\mathbf{J}_{j} \mathbf{r}_{j 3}
\end{aligned}
$$

The precise integration expression for modal responses is obtained by substituting Eqs (18) and (19) into Eq. (17). Furthermore, the response $\mathbf{u}\left(t_{k+1}\right)$ of the structure at time $t_{k+1}$ can be obtained as:

$$
\mathbf{u}\left(t_{k+1}\right)=\sum_{j=1}^{q} \phi_{j} z_{j}\left(t_{k+1}\right)
$$

The above expression has taken into account the variation of magnitude and position of the load, so that even if a very large step size is used, results can still be obtained to computer accuracy.

\section{Numerical examples}

The results presented are for the simply supported uniform beam and the three span non-uniform beam shown in Figs 3 and 4, respectively, in which $E I$ is flexural rigidity and $\bar{m}$ is mass per unit length. They are divided, respectively, into 10 and 50 Bernoulli-Euler beam elements of identical length. Note that although the examples are restricted to beams for convenience, the method presented applies to any structure which can be analyzed by FEM.

100 frequency points were used for all the examples which follow because this gave sufficient precision for the integration of Eq. (9a). The adequacy of this precision was verified because using 500 frequency points for each example gave results which were always within $1 \%$ of those given by 100 frequency points. 


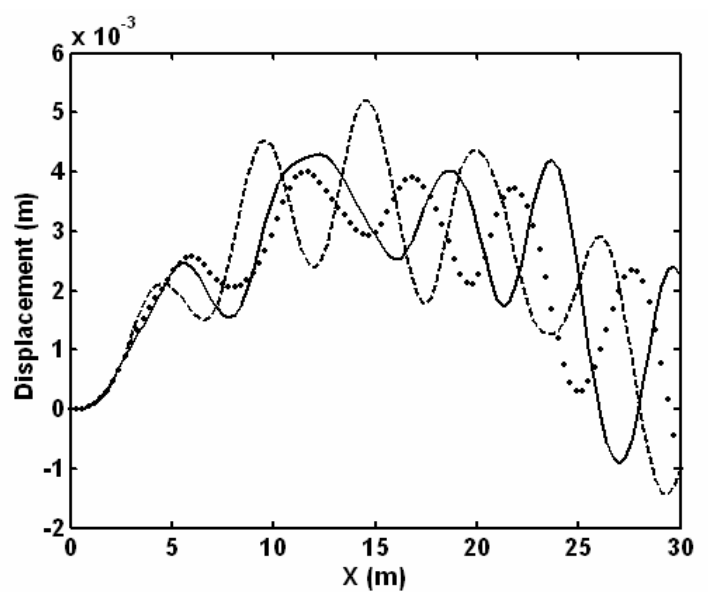

Fig. 5. The time histories of the displacement of the midpoint caused by different load samples.

\section{Example 1: Verifies the present method}

Assume that a single random load $p(t)$ with mean value $\bar{p}=500 \mathrm{kN}$ and velocity $V=100 \mathrm{~km} / \mathrm{hcrosses}$ the simply supported beam. This load has PSD function $S_{p}(\omega)=63.66 \mathrm{kN}^{2} / \mathrm{rad} / \mathrm{s}$ with $\omega \in[0,25 \pi]$, from which the standard deviation can be calculated as $\sigma_{p}=70.7 \mathrm{kN}$. The sample functions of the load are simulated by means of trigonometric series. Figure 5 gives three curves for the midpoint displacement caused by different load samples and it can be seen that they deviate from one another by quite large amounts. Figure 6 gives the standard deviation curves computed by the present PEM-based method and by the Monte Carlo simulation (MCS) with 1000 and 10000 samples, respectively. It can be seen that the results using the present method and MCS are in good agreement, and this agreement is improved by increasing the number of samples in the simulation. In the MCS procedure, the Newmark method is used and the sample functions of the random loads are simulated by the trigonometric series

$$
p(t)=\sqrt{2} \sum_{k=1}^{N} \sqrt{S_{p}\left(\omega_{k}\right) \Delta \omega} \cos \left(\omega_{k} t+\phi_{k}\right)
$$

where: $\Delta \omega$ is the frequency interval; $N$ is the total number of frequency intervals and; $\phi_{k}$ is a random variable uniformly distributed between 0 and $2 \pi$.

Ref. [2] gives the analytical solution for standard deviation of the midpoint displacement of a simply supported beam for a single moving load with the statistical characteristics $R_{p}(\tau)=\sigma_{p}^{2}$ and $S_{p}(\omega)=\sigma_{p}^{2} \delta(\omega)$. Figure 7 compares the standard deviation curves given by the present method, using only the fundamental mode, with these analytical ones, where $x$ represents the location of the load, $\sigma_{p}=100 \mathrm{kN}$ and $V=100 \mathrm{~km} / \mathrm{h}$. The excellent agreement between the two methods acts as a good check on the method presented and on the computer program developed from it.

\section{Example 2: Shows the efficiency and precision of the present method}

The main computational effort of the present method is that needed to compute the responses of a structure subjected to moving harmonic loads with different frequencies. These responses are independent of each other, so the total CPU time is approximately proportional to the number of harmonic terms used. Therefore, comparing the efficiency and precision for the response due to one harmonic term is sufficient to show the advantage of the present method. Table 1 compares the efficiency and precision of PIM with the Newmark method by virtue of the maximum midpoint displacement when a single load $100 \sin (\omega t) \mathrm{kN}$ with $\omega=25 \pi$ moving along the simply supported beam with velocity $V=100 \mathrm{~km} / \mathrm{h}$ and only the fundamental mode was used. A Pentium 4 computer with main frequency $1.8 \mathrm{GHz}$ and memory $256 \mathrm{M}$ was used and the analytical solution shown in the Table and used to calculate its error column was obtained from Ref. [1]. Whenever, as in this case, the FEM model of a structure is such that the elements 


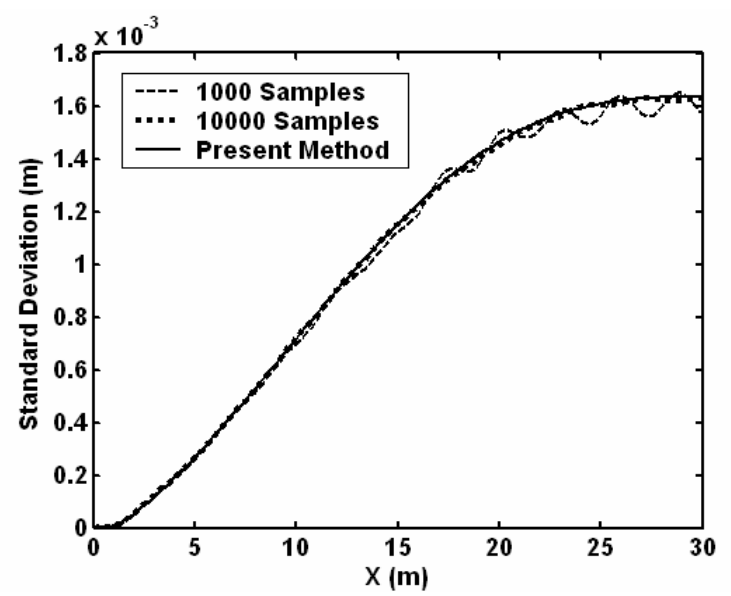

Fig. 6. Standard deviation curves computed by the present PEM-based method and by the Monte Carlo simulation with 1000 and 10000 samples.

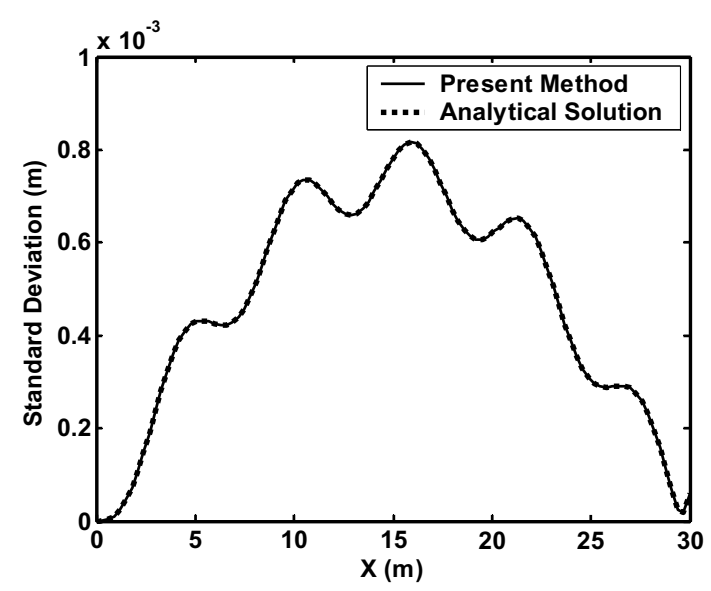

Fig. 7. The standard deviation of the midpoint deflection of the simply supported beam subjected to a load with PSD function $S_{p}(\omega)=\sigma_{p}^{2} \delta(\omega)$, with $\sigma_{p}=100 \mathrm{kN}$.

into which the beam(s) traversed by the load are of equal length it is possible to choose the integration step of PIM such that it divides exactly into the element length, so that condition 1 is satisfied at all integration steps and hence the answer is exact. This was done for the first three PIM results in the Table, for which the number of integration steps needed to traverse one element is, respectively, one, four and twelve. Thus all three results are identical, being the exact value for the FEM model used, i.e. 0.13434856 . Thus the tiny $0.000774 \%$ error shown for this result is entirely accounted for by the FEM discretisation errors.

The results show that if good accuracy is required, PIM is much more efficient that the Newmark method, e.g. if an error below $1 \%$ is required the Newmark method takes 0.049 CPU seconds, whereas PIM needs only 0.003 seconds while also giving computer precision. The fourth PIM result in the Table, i.e. when the integration step was $0.621 \mathrm{~s}$, was included because 5.5 elements were traversed in a single time step and so this forms an extreme test of the loss of accuracy when condition II is used, the loss being less than $0.08 \%$ for this case, which is very acceptable.

\section{Example 3: Shows the random vibration characteristics of the simply supported beam}

Figure 8 gives the displacement PSD of the midpoint, from $0 \mathrm{~Hz}$ to $10 \mathrm{~Hz}$ (the responses from $10 \mathrm{~Hz}$ to $25 \mathrm{~Hz}$ were relatively small), when a single load $p(t)$ crosses the beam with one of the three constant velocities shown. 
Table 1

Comparison of efficiency and precision between PIM and the Newmark method for the simply supported beam. (The maximum midpoint displacement occurs when the load is approximately $17.25 \mathrm{~m}$ from the left-hand end)

\begin{tabular}{lcccc}
\hline Method & $\begin{array}{c}\text { Integration } \\
\text { step } \\
(\mathrm{s})\end{array}$ & $\begin{array}{c}\text { Maximum } \\
\text { midpoint } \\
\text { displacement } \\
\times 10^{-3}(\mathrm{~m})\end{array}$ & $\begin{array}{c}\text { Error } \\
(\%)\end{array}$ & $\begin{array}{c}\text { CPU time } \\
(\mathrm{s})\end{array}$ \\
\hline \multirow{4}{*}{ Newmark } & 0.0270 & 0.042865413 & $68.1 \%$ & 0.005 \\
& 0.0090 & 0.12203824 & $9.16 \%$ & 0.015 \\
& 0.0054 & 0.12985657 & $3.34 \%$ & 0.024 \\
& 0.0027 & 0.13321921 & $0.841 \%$ & 0.049 \\
\hline \multirow{4}{*}{ PIM } & 0.1080 & & & 0.003 \\
& 0.0270 & 0.13434856 & $0.000774 \%$ & 0.010 \\
& 0.0090 & & & 0.031 \\
\hline Analytical & 0.6210 & 0.13445484 & $0.078 \%$ & $*$ \\
\hline
\end{tabular}

*Too small to measure.

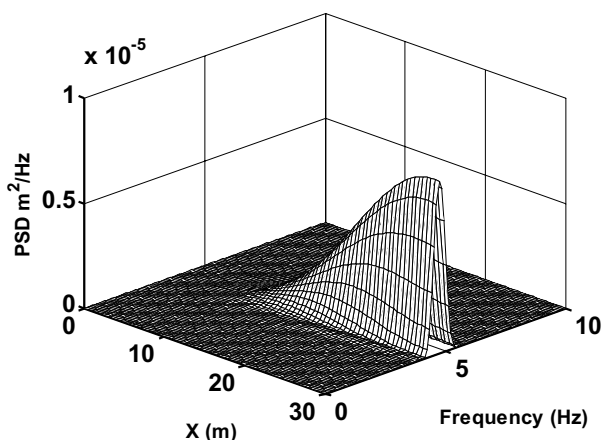

(a) $V=50 \mathrm{~km} / \mathrm{h}$

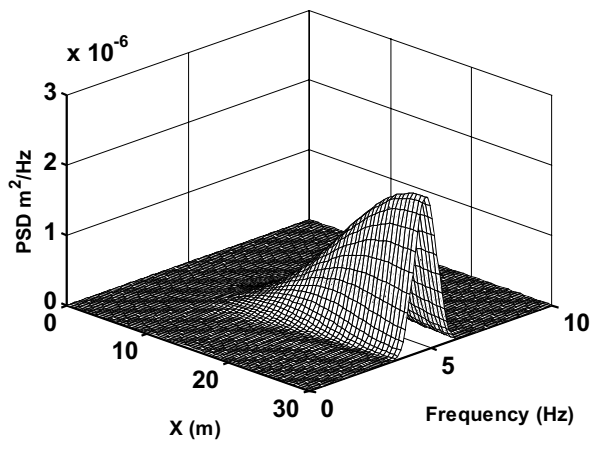

(b) $V=100 \mathrm{~km} / \mathrm{h}$

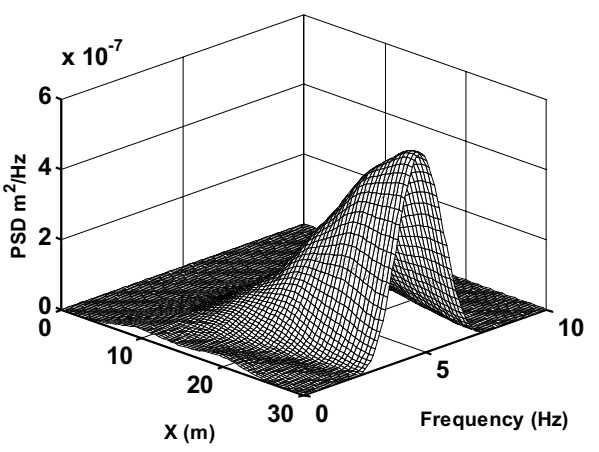

(c) $V=200 \mathrm{~km} / \mathrm{h}$

Fig. 8. The midpoint displacement PSD of the simply supported beam subjected to a load with velocity $V:$ (a) $V=50 \mathrm{~km} / \mathrm{h}$; (b) $V=100 \mathrm{~km} / \mathrm{h}$; (c) $V=200 \mathrm{~km} / \mathrm{h}$

The load has PSD function $S_{p}(\omega)=63.66 \mathrm{kN}^{2} / \mathrm{rad} / \mathrm{s}, \omega \in[0,50 \pi]$, i.e. its standard derivation is $\sigma_{p}=100 \mathrm{kN}$ and the solution used five modes and a damping ratio of 0.002 .

It can be seen that the response peaks always appear around the fundamental frequency $4.77 \mathrm{~Hz}$ of the beam and decrease rapidly with increasing velocity. This is logical because at higher load velocities the excitation time is shorter and hence there is less time for the beam response to develop. 


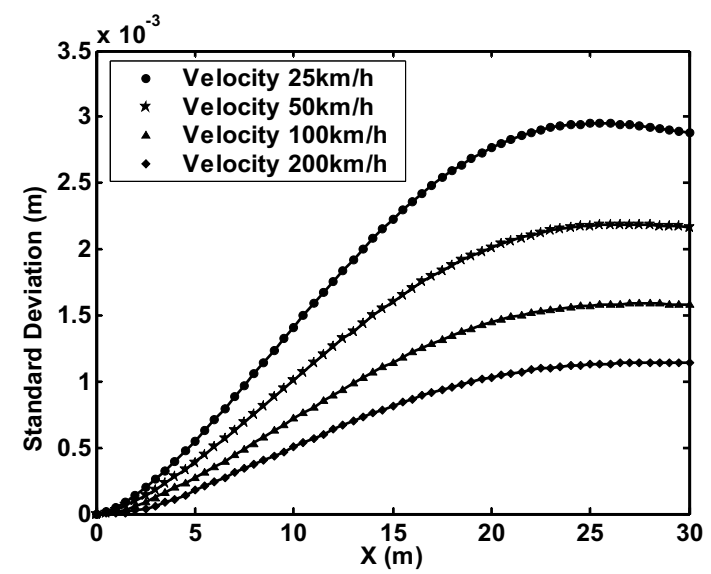

Fig. 9. The standard deviation of the midpoint deflection of the simply supported beam subjected to loads with different velocities. The solid lines are for full correlation and the symbols are the results without correlation.

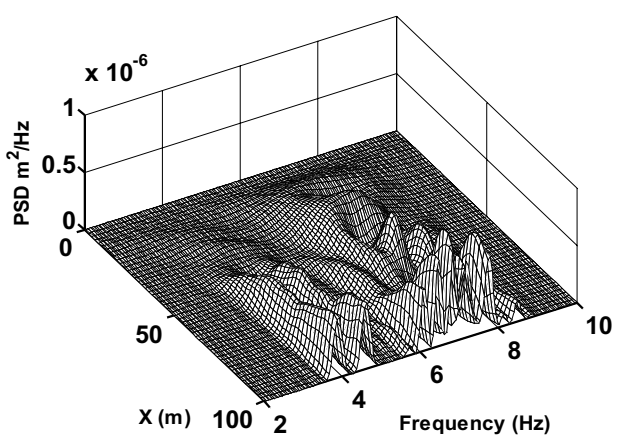

(a) Left-hand span (right-hand span results are similar)

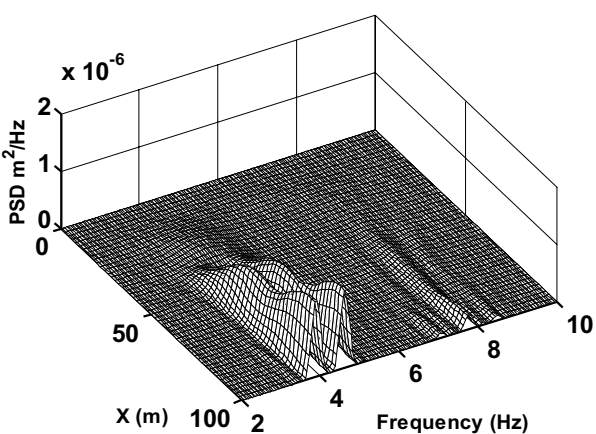

(b) Central span

Fig. 10. The midpoint displacement PSD's for the three spans of the non-uniform beam. (a) Left-hand span (right-hand span results are similar), (b) Central span.

Figure 9 shows the standard deviations of the midpoint and compares the results with those obtained by ignoring the correlation between the modal responses. Clearly correlation has insignificant influence for this example. Moreover, it can be seen that the maximum response decreases with increasing velocity. Ref [2] gives similar results for white noise loading. Generally, the value of maximum response varies with the velocity and type of load and Ref. [2] gives the values corresponding to 5 types of random loads traveling at various speeds in order to demonstrate this phenomenon.

\section{Example 4: Shows the random vibration characteristics of the three span beam}

The problem is identical to that of Example 3 except that now it is for the three span beam and so 10 modes were used and the first three natural frequencies were $4.25 \mathrm{~Hz}, 6.65 \mathrm{~Hz}$ and $7.77 \mathrm{~Hz}$.

Figure 10 shows the midpoint displacement PSD of the three spans for $V=100 \mathrm{~km} / \mathrm{s}$. It can be seen that the PSD for the central span is concentrated around the fundamental frequency of the beam whereas for the outer spans the high PSD values approximately span its first three natural frequencies.

Figure 11 compares the standard deviation curves with those obtained by ignoring the correlation between the modal responses. Clearly correlation has insignificant influence for the central span of this example but has considerable effect for the outer spans.

$\mathrm{Li}$ [19] studied the influence of the cross-correlation between modal responses in the stationary random vibration analysis of structures and pointed out that if the ratio between the main participating natural frequencies is lower 


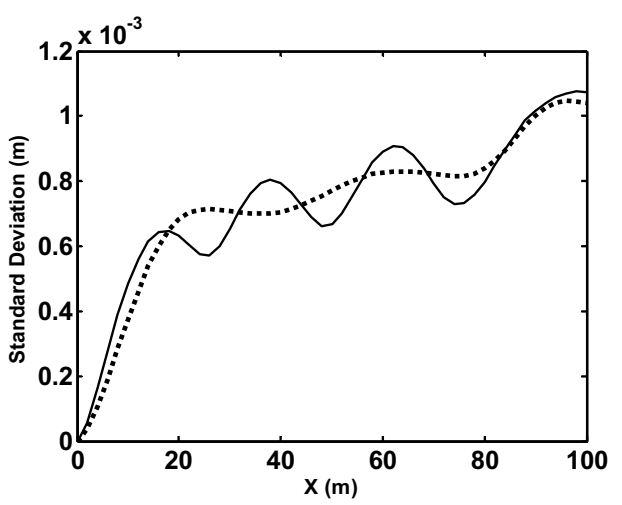

(a) Left-hand span(right-hand span results are similar)

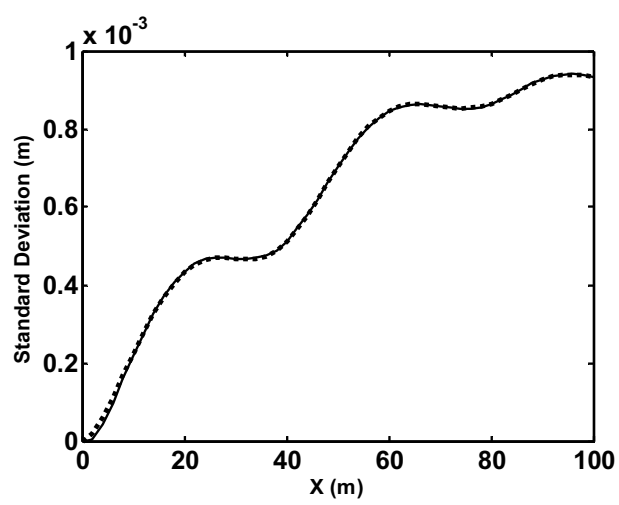

b) Central span

Fig. 11. The standard deviations for midpoint deflections of the three spans of the non-uniform beam. Key: Full correlation; without correlation. (a) Left-hand span (right-hand span results are similar), (b) Central span.

than 1:3, the influence of the correlation is significant, whereas otherwise it is insignificant. From Figs $8-11$ it can be seen that for the non-stationary vibration of beams, although the threshold ratio is not 1:3, the influence of the correlation is still related to the ratio between the main participating natural frequencies, as follows.

For the simply supported beam and for the central span of the three span beam the second mode is anti-symmetric and so only the first and third modes can make significant contributions to the midpoint response, for which the component from the first mode was observed to be much larger than that from the third mode. Since the ratio of the third and first natural frequencies is not very close to unity (it is $7.77 \mathrm{~Hz} / 4.25 \mathrm{~Hz}=1.83$ for the three span beam), this accords with the conclusion stated at the end of the previous paragraph. In contrast, for the outer spans, the contributions of the first three modes at the midpoint were observed to be of about the same level, while the ratios between the successive frequency pairs are $6.65 / 4.25=1.56$ and 7.77/6.65 $=1.17$, respectively, which are comparatively closer to unity. This again accords with the conclusion stated at the end of the previous paragraph.

\section{Example 5: Shows the influence of correlation between loads}

The problem is identical to the simply supported beam one of Example 3 except that now a second, identical, load crosses the beam such that the loads are $d$ apart and move with velocity $V$, for the three combinations of $V$ and $d$ shown in Fig. 12, which gives the standard deviation of the midpoint displacement. Five modes and a damping ratio 0.002 were used for computation. The two cases considered are those of the loads being either fully correlated or independent, so that for the former Eq. (5) results in $a_{i}=1$ and $t_{i}=d / V$, for $i=1,2$.

It can be seen that for $d=5 \mathrm{~m}$ and $V=50 \mathrm{~km} / \mathrm{h}$, or for $d=8$ mand $V=100 \mathrm{~km} / \mathrm{h}$, the responses are higher when the correlation is ignored, whereas they are lower for $d=5 \mathrm{~m}$ and $V=100 \mathrm{~km} / \mathrm{h}$. Such discrepancies between whether the correlation (which can be regarded as the effects between the items $a_{j} e^{-i \omega t_{j}}, j=1,2, \cdots l$ in Eq. (7b)) increases or decreases the responses are to be expected because the randomness of the phases of the two correlated loads causes their effects either to reinforce one another or to tend to cancel one another. As all $t_{j}$ are constants, the inclusion of such correlation items will not increase the computation time when using PEM.

\section{Example 6: Shows the influence of different types of load}

The problem is a single load with any of statistical characteristics given as (A)-(C) below which crosses the simply supported beam with velocity $V=100 \mathrm{~km} / \mathrm{h}$.

$$
\begin{aligned}
& \text { (A) } S_{p}(\omega)=\sigma_{p}^{2} / \Delta \omega, \Delta \omega=30 \pi, \omega \in[0,30 \pi] \\
& \text { (B) } S_{p}(\omega)=2 \sigma_{p}^{2} \omega_{\mathrm{g}} /\left(\omega^{2}+\omega_{g}^{2}\right) / \pi, \quad \omega_{g}=5, \omega \in[0,30 \pi]
\end{aligned}
$$




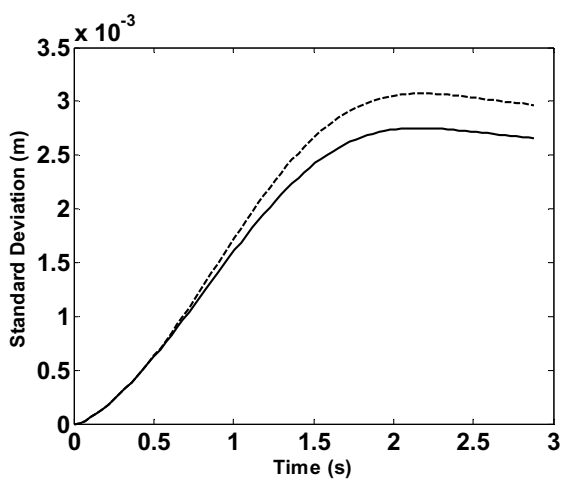

(a) $d=5 \mathrm{~m}, \quad V=50 \mathrm{~km} / \mathrm{h}$

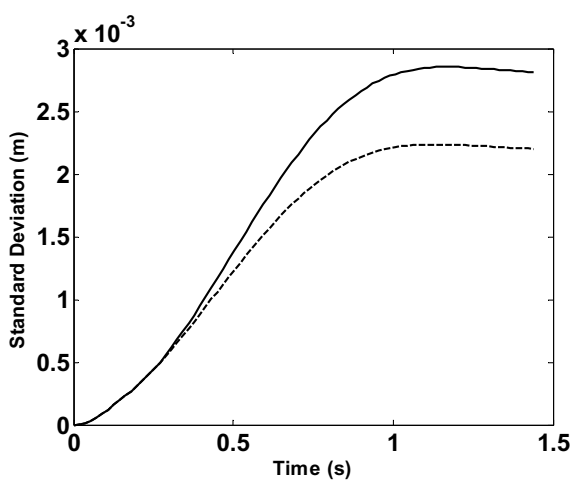

(b) $d=5 \mathrm{~m}, \quad V=100 \mathrm{~km} / \mathrm{h}$

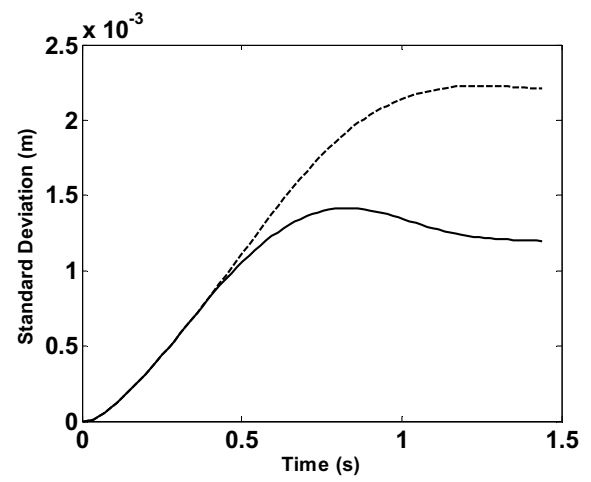

(c) $d=8 \mathrm{~m}, \quad V=100 \mathrm{~km} / \mathrm{h}$

Fig. 12. The standard deviation of the midpoint deflection of the simply supported beam when subjected to two identical loads which were $d$ apart. Key: full correlation; without correlation. (a) $d=5 \mathrm{~m} £<V=50 \mathrm{~km} / \mathrm{h} ;$ (b) $d=5 \mathrm{~m} £<V=100 \mathrm{~km} / \mathrm{h} ;$ (c) $d=8 \mathrm{~m} £<V=100 \mathrm{~km} / \mathrm{h}$.

(C) $\begin{aligned} & S_{p}(\omega)=\sigma_{p}^{2} \omega_{\mathrm{g}}\left[1 /\left\{\omega_{g}^{2}+\left(\omega+\omega_{0}\right)^{2}\right\}+1 /\left\{\omega_{g}^{2}+\left(\omega-\omega_{0}\right)^{2}\right\}\right] / \pi \\ & \omega_{\mathrm{g}}=2, \quad \omega_{0}=16 \pi, \quad \omega \in[0,30 \pi]\end{aligned}$

These PSD functions were integrated with respect to the frequency $\omega$ and assumed to have identical standard deviations $\sigma_{p}=100 \mathrm{kN}$, in order to obtain Figs 13 and 14, which respectively show the distribution of these PSD in the frequency domain and the standard deviation curves of the midpoint displacement.

The identical standard deviations mean that the areas under all of the PSD curves of Fig. 13 are equal. However Fig. 14 clearly shows that the responses vary with the shape of the PSD curves. In examples 3 and 4, it can be found that the response PSD peaks always appear in the range spanned by the main participating natural frequencies, i.e. if the peak of PSD is quite close to the dominant natural frequency of the structure, the response will be very large.

\section{Conclusions}

An accurate and efficient method has been proposed for the study of non-stationary random vibration of structures which are subjected to moving loads and are modeled by FEM. The loads are assumed to be stationary random processes with constant mean values from which PEM (the Pseudo Excitation Method) is used to derive nonstationary PSD's and time-dependent standard deviations. A consistent decomposition procedure has been presented to simulate the movement of the loads and the corresponding precise integration expression has been given. The numerical examples are for a simply supported beam and for a three span non-uniform beam. Their random dynamic characteristics have been investigated and result in the following conclusions: 


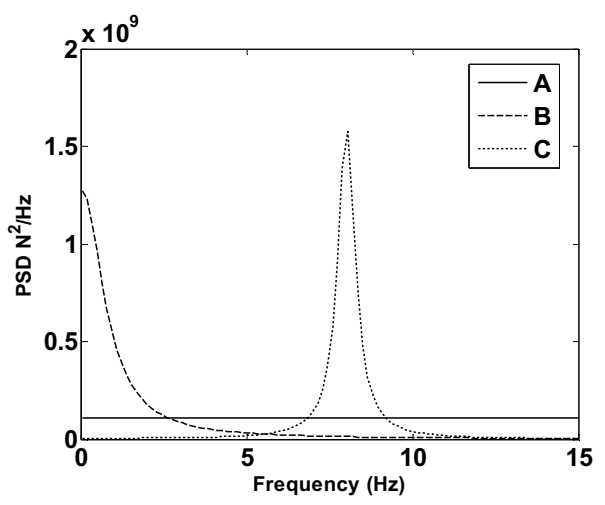

Fig. 13. The excitation PSD's of load types A, B and C for the simply supported beam.

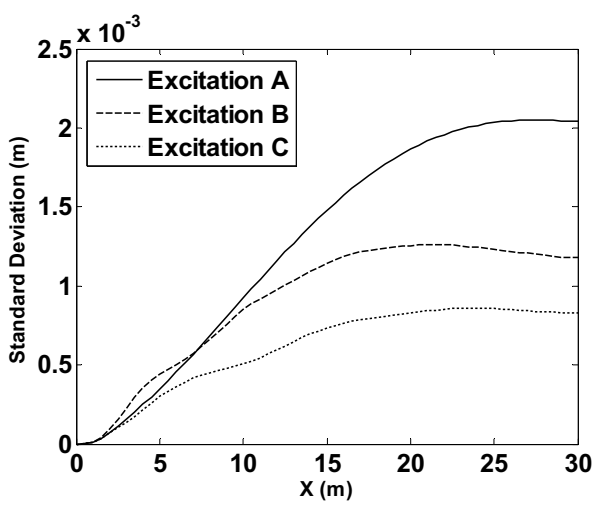

Fig. 14. The standard deviation of the midpoint deflections of the simply supported beam when subjected to load types A, B and C.

1. the non-stationary PSD's peak in the range spanned by the main participating natural frequencies;

2. if the ratios between the main participating natural frequencies are small correlation of modal responses is significant, whereas otherwise it is not;

3. when there is a chain of moving loads, the cross-correlation between them influences the response strongly;

4. The response is strongly affected by the type of load, even if the standard deviation is same in each case.

\section{Acknowledgements}

The authors are grateful for support from the National Natural Science Foundation of China (grant Nos 10472023, 10502011) and from the Cardiff Advanced Chinese Engineering Centre.

\section{References}

[1] L. Fryba, Vibration of Solids and Structures Under Moving Loads, Thomas Telford, London, 1999.

[2] L. Fryba, Non-stationary response of a beam to a moving random force, Journal of Sound and Vibration 46 (1976), $323-338$.

[3] R. Iwankiewicz and P. Sniady, Vibration of a beam under a random stream of moving forces, Journal of Structure Mechanics 12 (1984), 13-26.

[4] G. Ricciardi, Random vibration of beam under moving loads, Journal of Engineering Mechanics-ASCE 120 (1994), 2361-2380.

[5] R.T. Wang and T.Y. Lin, Random vibration of multi-span Timoshenko beam due to a moving load, Journal of Sound and Vibration 213 (1998), 127-138.

[6] P. Sniady, S. Biernat, R. Sieniawska and S. Zukowski, Vibrations of the beam due to a load moving with stochastic velocity, Probabilistic Engineering Mechanics 16 (2001), 53-59. 
[7] H.S. Zibdeh and R. Rackwitz, Response moments of an elastic beam subjected to Poissonian moving loads, Journal of Sound and Vibration 188 (1995), 479-495.

[8] H.S. Zibdeh, Stochastic vibration of an elastic beam due to random moving loads and deterministic axial forces, Engineering Structures 17 (1995), 530-535.

[9] H.S. Zibdeh and R. Rackwitz, Moving loads on beams with general boundary conditions, Journal of Sound and Vibration 195 (1996), 85-102.

[10] H.S. Zibdeh and H.S. Juma, Dynamic response of a rotating beam subjected to a random moving load, Journal of Sound and Vibration 223 (1999), 741-758.

[11] H.S. Zibdeh and M. Abu-Hilal, Stochastic vibration of laminated composite coated beam traversed by a random moving load, Engineering Structures 25 (2003), 397-404.

[12] M. Abu-Hilal, Vibration of beams with general boundary conditions due to a moving random load, Archive of Applied Mechanics 72 (2003), 637-650

[13] J.H. Lin, W.S. Zhang and J.J. Li, Structural responses to arbitrarily coherent stationary random excitations, Computers \& Structures $\mathbf{5 0}$ (1994), 629-633.

[14] J.H. Lin and Y.H. Zhang, Pseudo Excitation Method in Random Vibration, Science Press, Beijing, 2004. (in Chinese).

[15] J.H. Lin and Y.H. Zhang, Seismic Random Vibration of Long-span Structures, in: Vibration and Shock Handbook, C.W. de Silva, ed., CRC Press, Boca Raton, Florida, 2005.

[16] W.X. Zhong and F.W. Williams, A precise time step integration method, Proceedings of the Institution of Mechanical Engineers (208C) 1995, 427-430.

[17] W.X. Zhong, Duality System in Applied Mechanics and Optimal Control, Kluwer Academic, Boston, 2004.

[18] J.H. Lin, S.Y. Zhang, F. Lu and Y.H. Zhang, Precise integration for bridges subjected to moving harmonic loads, Chinese Journal of Computational Mechanics 23 (2006), 385-390 (in Chinese).

[19] G.H. Li, Earthquake-resistance Dynamics of Engineering Structures, Shanghai Press of Science and Technology, Shanghai, 1984 (in Chinese). 

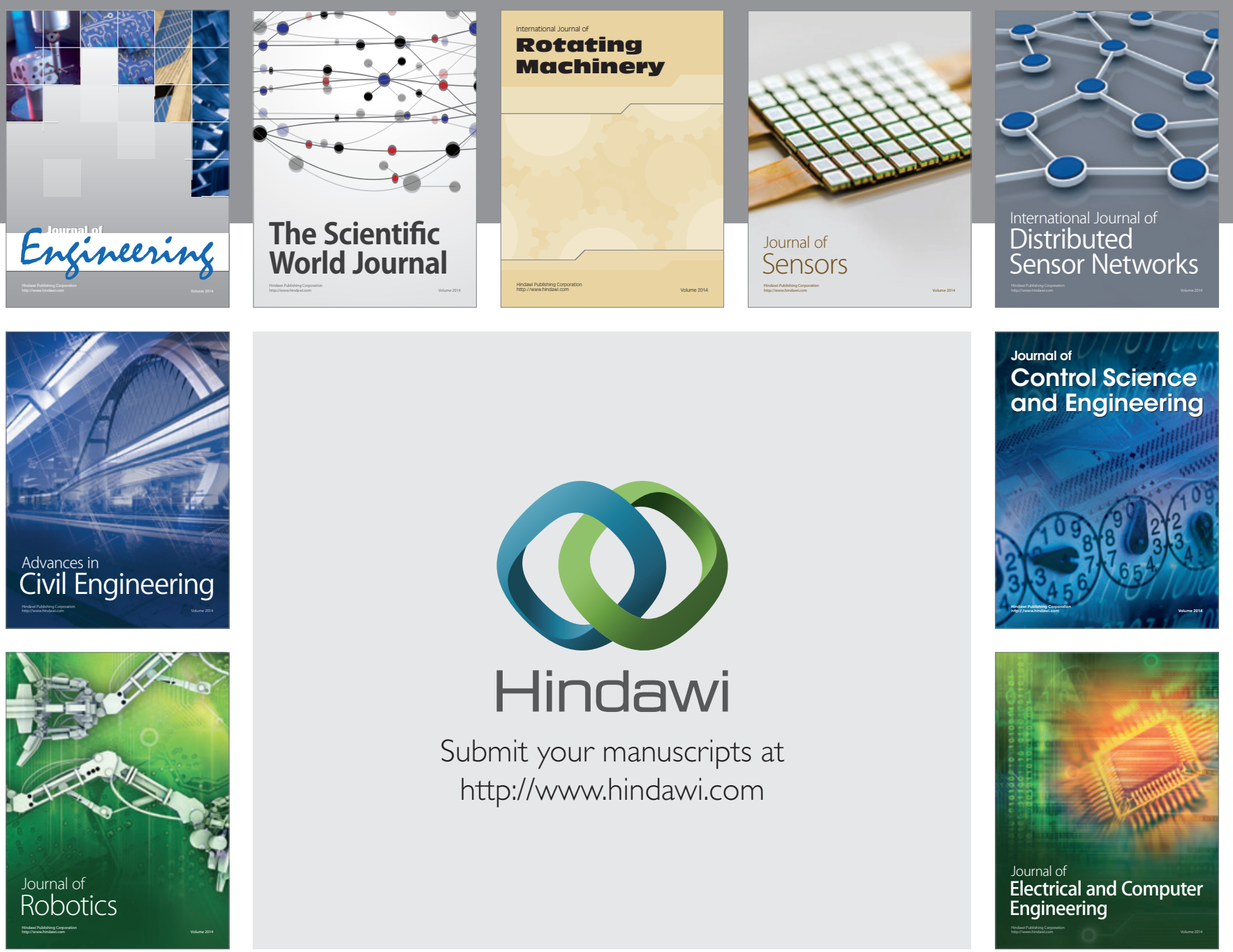

Submit your manuscripts at

http://www.hindawi.com
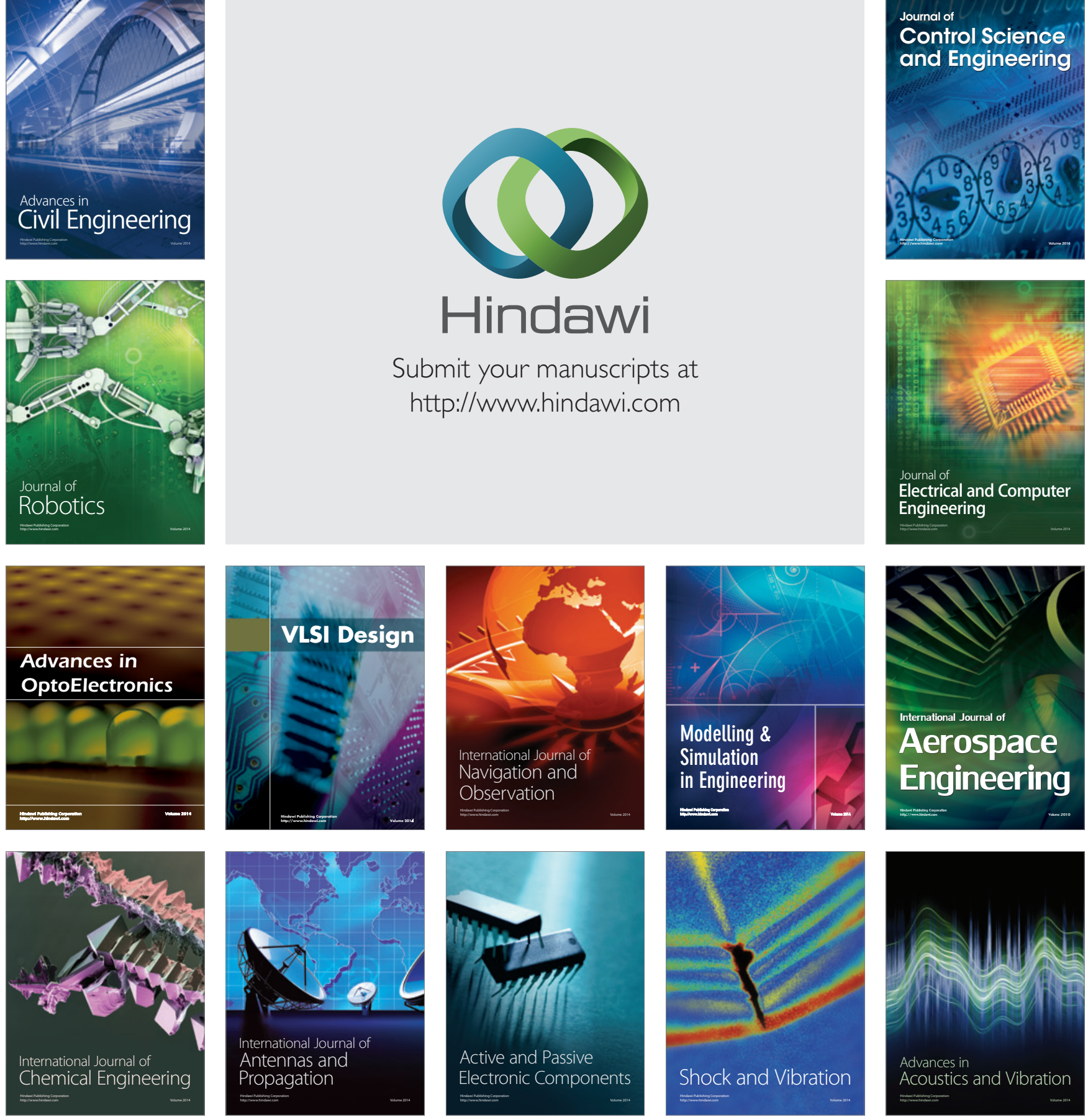